\title{
VALORES HUMANOS COMO EXPLICADORES DE ATITUDES AMBIENTAIS E INTENÇÃO DE COMPORTAMENTO PRÓ-AMBIENTAL
}

\author{
Jorge Artur Peçanha de Miranda Coelho* \\ Valdiney Veloso Gouveiä \\ Taciano Lemos Milfont ${ }^{\llbracket}$
}

\begin{abstract}
RESUMO. Os valores humanos têm sido apontados como importantes para o entendimento e predição de atitudes e comportamentos pró-ambientais. Embora a literatura saliente a relação destes três construtos, nenhuma informação foi encontrada considerando amostras brasileiras. Este estudo buscou contribuir para preencher esta lacuna. O estudo contou com a participação de 208 estudantes universitários, sendo a maioria de uma universidade privada (63,8\%), do sexo feminino $(73,4 \%)$ e com idade média de 28 anos $(D P=7,0)$. Estes responderam a dois instrumentos: Questionário dos Valores de Schwartz e Escala de Atitudes Ecocêntricas e Antropocêntricas, além de uma pergunta acerca da intenção de se engajar em comportamento pró-ambiental e questões demográficas. Os resultados corroboraram estudos prévios, indicando que os valores de autotranscendência, especificamente os de orientação universalista, predizem atitudes e comportamentos pró-ambientais. Os resultados são discutidos, propondo a utilização dos valores humanos como facilitadores na promoção de comportamentos em prol do ambiente.
\end{abstract}

Palavras-chave: valores humanos, atitudes ambientais, comportamento pró-ambiental.

\section{HUMAN VALUES AS PREDICTORS OF ENVIRONMENTAL ATTITUDES AND PRO-ENVIRONMENTAL BEHAVIOR}

\begin{abstract}
Human values have been pointed out as important predictors of environmental attitudes and pro-environmental behaviors. Although the literature supports the relationship between these three constructs, no information was found considering Brazilian samples. This study aimed to contribute to this gap. Participated in this study a number of 208 undergraduate students; most of them were from a private university (63.8\%), female (73.4\%) and with mean age of 28 years $(S D=7.0)$. They answered the Schwartz Values Survey and the Echocentric and Anthropocentric Attitudes Scale, an item to measure intention of pro-environment behaviors, and demographic questions. Results support previous studies. Specifically, self-transcendence values were the most important to explain pro-environmental attitudes and behaviors. These findings are discussed regarding the possibility of using human values in interventions to promote behaviors on behalf of the environment.
\end{abstract}

Key words: Human values, environmental attitudes, pro-environmental behavior.

Após a Revolução Industrial, os padrões interativos do ser humano com o meio ambiente caracterizaram-se pela busca de ganhos socioeconômicos. (Almeida Junior, 1994). O aquecimento global, a destruição da camada de ozônio, o desmatamento, a extinção de espécies, a diminuição do suprimento de água potável, o crescimento populacional, a chuva ácida e a poluição tóxica do ar e das águas vêm sendo apontados como as principais ameaças ao meio ambiente terrestre (Elston, 2001; Oskamp, 2000).

Doze milhões de pessoas de todo o mundo morrem por ano por não terem água potável (Hinrichsen \& Robey, 2000). Acrescente-se que cerca da metade das florestas originais do mundo foi perdida (todo ano quase 40 milhões de acres são cortados ou queimados) e que duas de cada três espécies ainda vivas estão em processo de extinção (Elston, 2001). Anualmente 30 bilhões de toneladas de lixo são

\footnotetext{
* Mestre em Psicologia Social pela Universidade Federal da Paraíba. Professor da Faculdade Associação Paraibana de Ensino Renovado-ASPER.

\# Doutor pela Universidad Complutense de Madrid, Espanha. Professor Adjunto da Universidade Federal da Paraíba.

II Doutorando da University of Auckland, Nova Zelândia. Professor Assistente da Universidade Federal de Alagoas.
} 
despejados na natureza em todo o mundo (no Brasil são produzidas diariamente 125.281 toneladas). Em menos de vinte anos, o volume de água distribuída sem tratamento aos brasileiros aumentou mais de 3\%, indo de 3,9\% em 1989 para 7,2\% em 2000 (Alves Filho, 2002). Por estes motivos, a Organização das Nações Unidas (ONU) espera que $20 \%$ da população mundial se transformem em "refugiados ambientais" nos próximos 20 anos, devido aos danos ambientais causados em suas áreas de origem, tais como a destruição de terra fértil e carência de água (Oskamp, 2000).

Datam da década de 1970 as primeiras discussões da comunidade internacional sobre os limites do desenvolvimento do planeta, quando começaram a ser apontados os riscos da degradação do meio ambiente (o primeiro relatório científico sobre os danos para a camada de ozônio foi publicado em 1974; Oskamp, 2000). Tais discussões ganharam tamanha intensidade que levaram a ONU a promover uma Conferência sobre o Meio Ambiente, realizada em Estocolmo em 1972. A partir das constatações científicas de que os recursos da Terra não são inesgotáveis e que a preservação do ecossistema está intimamente relacionada com a preservação da espécie humana, movimentos ambientalistas, organizações governamentais e nãogovernamentais vêm discutindo a necessidade de serem promovidos padrões sustentáveis de crenças, valores, atitudes e comportamentos, pois as riquezas naturais estão sendo consumidas em ritmo muito mais acelerado do que sua capacidade de regeneração.

Este antropocentrismo tradicional, no qual se faz presente a percepção do "dominador" (ser humano) versos "dominado" (natureza) é atribuído a múltiplos fatores históricos, do tipo: a expansão européia durante o século XVI, com a "descoberta" de novos mundos com recursos aparentemente inesgotáveis; o predomínio de cristãos convictos da dominação dos homens sobre outras criaturas (ver Schultz, Zelezny \& Dalrymple, 2000); o florescimento do capitalismo; o desenvolvimento das capacidades científicas e tecnológicas, e a dedicação ao contínuo aperfeiçoamento e progresso da sociedade humana (Dunlap \& Van Liere, 1978). Este paradigma antropocêntrico caracteriza uma crença no não-esgotamento dos recursos naturais, progresso contínuo e necessidade de desenvolvimento; expressa uma confiança na resolução de problemas pela ciência e tecnologia e um forte compromisso com a economia de livremercado e propriedade privada (Albrecht, Bultena, Hoiberg \& Nowak, 1982).
Os problemas ambientais podem ser entendidos como causados por comportamentos humanos maladaptados (Maloney \& Ward, 1973), e por isso a Psicologia tem sido apontada como detentora de um importante papel na minoração de tais problemas (Cortez, Milfont \& Belo, 2001; Gouveia, 2002; Howard, 2000; Kidner, 2001; McKenzie-Mohr, 2000; Oskamp, 2000; Stern, 2000; Weigel \& Weigel, 1978).

Assim, o estudo dos valores e das atitudes, bem como suas modificações, pode contribuir consistentemente para oferecer intervenções que possibilitem a modifição comportamental. Por exemplo, pesquisas anteriores apontam a importância dos valores e das atitudes para a promoção de comportamentos próambientais (Bechtel, Verdugo \& Pinheiro, 1999; Dunlap \& Van Liere, 1978; Schultz \& Zelezny, 1999; Stern \& Dietz, 1994; Stern, Dietz \& Kalof, 1993; Thompson \& Barton, 1994; Weigel \& Weigel, 1978). Não obstante, são escassas as informações a respeito de valores e atitudes ambientais em que tenham sido consideradas amostras brasileiras. Nesse sentido, objetivou-se conhecer o papel dos valores humanos como explicadores das atitudes ambientais e da intenção comportamental de universitários em agir em prol do meio ambiente. Faz-se necessário, previamente, considerar o marco teórico de referência, definindo os construtos aqui tratados: valores, atitudes e comportamentos pró-ambientais.

\section{VALORES HUMANOS}

Pesquisas em Psicologia Social que buscam explicar as relações das atitudes e comportamentos pró-ambientais têm utilizado dois principais marcos teóricos: (1) as teorias da escolha racional [Rationalchoise theories, tal como a Teoria da ação racional (Ajzen \& Fishbein, 1985)], e (2) a Teoria da ativação da norma (Schwartz, 1977). De acordo com a Teoria da ação racional, o comportamento é determinado pela intenção de executar um comportamento pró-ambiental (ou outro comportamento qualquer) concreto. Esta intenção, por sua vez, é determinada pela atitude sobre o comportamento (componente atitudinal), pela norma subjetiva (componente normativo) e pelo controle percebido do sujeito sobre o comportamento. Já a Teoria da ativação da norma busca explicar os mecanismos que levam uma pessoa a agir de maneira altruísta. Este comportamento altruísta depende da ativação de normas pessoais (obrigação moral), e esta ativação depende dos valores do indivíduo.

Pesquisas têm demonstrado que a Teoria da ação racional tem falhado em predizer comportamentos 
moralmente relacionados, como é o caso dos próambientais, enquanto a Teoria da ativação da norma e suas derivações predizem mais adequadamente tais comportamentos, por contemplar mais eficazmente o domínio moral (Kaiser, Ranney, Hartig \& Bowler, 1999). Sendo assim, utilizou-se este último postulado teórico neste estudo, centrando-se especificamente no construto dos valores humanos. Nos últimos anos, vários estudos têm sido realizados relacionando os valores com questões ambientais. $\mathrm{O}$ fundamento deste embasamento teórico tem sido proporcionado principalmente por Shalom H. Schwartz, primeiro através da sua teoria da ativação da norma e, logo, com a proposta dos tipos motivacionais de valores (García-Mira, Real-Deus \& Romay-Martínez, 2000). Como comentam Schultz e Zelezny (1999), cada vez mais pesquisas são realizadas com o objetivo de tentar predizer as atitudes ambientais a partir dos valores.

Em linhas gerais, os valores humanos são aqui compreendidos como importantes metas ou normas que servem como princípios-guia na vida das pessoas (Rokeach, 1968/1981; Schultz \& Zelezny 1999). Embora outros modelos orientados à predição do comportamento ecológico responsável tenham sido propostos, derivados do modelo de Schwartz (Stern \& Dietz, 1994; Stern, Dietz \& Kalof, 1993), este trabalho se centrou concretamente na teoria dos tipos motivacionais dos valores humanos (Schwartz, 1992, 1994; Schwartz \& Bilsky, 1987, 1990). Esta é uma teoria que trata de dois dos aspectos principais dos tipos motivacionais: (1) conteúdo: propõe que os valores específicos (por exemplo, obediência, polidez) se reúnem em regiões ou espaços que conformam tipos motivacionais (por exemplo, tradição); e (2) Estrutura: indica que os tipos motivacionais se organizam de modo a formar um círculo, onde aqueles mais compatíveis estarão próximos (por exemplo, realização e poder), situando-se em lados opostos ou afastados daqueles com os quais são incompatíveis (por exemplo, conformidade e tradição).

A teoria de Schwartz (1992) propõe a existência de dez tipos motivacionais de valores. As relações estruturais entre os valores e os tipos motivacionais podem ser sintetizadas através de duas dimensões bipolares: abertura à mudança versus conservação e autopromoção versus autotranscendência. A primeira dimensão refere-se à tendência das pessoas a seguir seus interesses ou a se manter conforme as normas sociais, enquanto a segunda refere-se à motivação das pessoas para promover seus interesses, mesmo em detrimento dos outros, ou transcender os interesses pessoais, promovendo o bem-estar dos outros e da natureza (Schwartz, 1992).

Este modelo tem sido considerado como marco de referência em diversos estudos no Brasil há pelo menos uma década (Tamayo, 1994, 1997; Tamayo \& Schwartz, 1993), reunindo provas de sua adequação (Gouveia, Martínez, Meira \& Milfont, 2001). Pesquisas recentes têm utilizado este modelo para explicar, além das atitudes e comportamentos próambientais, o uso de preservativo (Tamayo, Lima, Marques \& Martins, 2000), o trabalho de equipe (Dutra \& Tamayo, 2000), a exaustão emocional (Tamayo, 2001; Tamayo, Lima, Siqueira, Vargas, Salomão \& Rodrigues, 2000; Tamayo, Pereznebra, Zandonadi \& Benjamin, 2002), a comparação entre os valores de presidiários e agentes penitenciários (Tamayo, Zandonadi, Nepomuceno, Costa Filho \& Barros, 2000) e o comprometimento organizacional (Tamayo, Souza, Vilar, Ramos, Albenaz \& Ferreira, 2001; Zandonadi, Pérez \& Tamayo, 2001).

\section{ATITUDES AMBIENTAIS}

As atitudes podem ser definidas sucintamente como "uma organização duradoura de crenças e cognições em geral, dotada de carga afetiva pró ou contra um objeto social definido, que predispõe a uma ação coerente com as cognições e afetos relativos a este objeto" (Rodrigues, Assmar, \& Jablonski, 1999, p. 100). As atitudes ambientais podem ser consideradas como sentimentos favoráveis ou desfavoráveis acerca do meio ambiente ou sobre um problema relacionado a ele, e têm sido definidas como as "percepções ou convicções relativas ao ambiente físico, inclusive fatores que afetam sua qualidade (por exemplo, superpopulação, poluição)" (American Psychological Association, 2001, p. 89). Tais atitudes podem se referir a experiências subjetivas e aprendidas, apresentando em sua composição as crenças relacionadas ao objeto atitudinal (nesta pesquisa, o meio ambiente) e sendo expressas através do comportamento (Hernández \& Hidalgo, 1998). Estas atitudes, como argumentam e demonstram Weigel e Newman (1976), correlacionam-se de forma significativa com índices de comportamento próambiental.

Tradicionalmente, os estudos sobre atitudes ambientais têm se centrado principalmente nos aspectos relativos à poluição, população e recursos naturais (Dunlap \& Van Liere, 1978), além de considerarem atitudes e comportamentos específicos, tais como reciclagem, consumismo e conservação de energia e água (Kaiser, Ranney, Hartig \& Bowler, 1999). Não obstante, Thompson e Barton (1994) desenvolveram uma medida para avaliar atitudes pró- 
ambientais mais gerais. Estes autores propõem a existência de dois tipos de atitudes ambientais: ecocêntricas e antropocêntricas. Ambas expressam preocupação ambiental e interesse em preservar a natureza e seus recursos; porém, os motivos desencadeadores dessa preocupação e interesse ambiental são distintos.

$\mathrm{O}$ antropocentrismo tem como base motivacional o interesse em manter a qualidade de vida, a saúde e a existência humana, e, para tanto, faz-se necessário preservar os recursos naturais e o ecossistema; havendo assim uma relação de troca, em que o homem preserva a natureza para seu benefício. Já para o ecocentrismo, a natureza é uma dimensão espiritual e de valor intrínseco que é refletida nas experiências humanas relacionadas com os sentimentos sobre o ambiente natural; o homem está conectado à natureza e a valoriza por si mesma. Em outras palavras, atitudes antropocêntricas estão baseadas nos efeitos que os problemas ambientais estão causando nos seres humanos, enquanto as ecocêntricas se baseiam em valores intrínsecos da natureza (Schultz, 2002).

\section{COMPORTAMENTO PRÓ-AMBIENTAL}

O comportamento pró-ambiental tem sido um dos temas de maior interesse em Psicologia Ambiental (Corral-Verdugo \& Pinheiro, 1999), e pode ser definido como "O conjunto de ações dirigidas, deliberadas e efetivas que respondem a requerimentos sociais e individuais e que resultam na proteção do meio" (Corral-Verdugo, 2000, p. 471).

Hines, Hungerford e Tomera (1987) realizaram uma meta-análise para identificar, analisar e sintetizar as variáveis identificadas em estudos prévios (entre 1971 e 1987) que apresentaram associação com comportamentos pró-ambientais. Foram analisadas 128 pesquisas, as quais foram divididas em quatro grupos segundo suas variáveis preditivas: (1) variáveis cognitivas (conhecimento), (2) variáveis psicossociais (atitudes, locus de controle, comprometimento verbal, responsabilidade pessoal e orientação econômica), (3) variáveis demográficas (nível educacional, renda, sexo e idade), e (4) uma categoria de pesquisas experimentais. Comprometimento verbal foi a variável que apresentou a maior relação $(r=0,49)$, enquanto sexo apresentou a menor $(r=0,08)$. Embora não tenham apresentado resultados sobre os valores humanos, vale ressaltar que a terceira variável mais importante foi atitude ambiental ( $r=$ $0,35)$.
Existem diversas escalas para avaliar comportamentos pró-ambientais, tais como a(s) proposta(s) por Schultz (Schultz \& Zelezny, 1999; Schultz, Zelezny, \& Dalrymple, 2000) e Kaiser (1998). O presente estudo utilizou, no entanto, uma medida que avaliou apenas a intenção comportamental, como detalhado no método. Este é um procedimento comum utilizado para a mensuração de tais comportamentos (ver, por exemplo, Cameron, Brown \& Chapman, 1998).

\section{VALORES, ATITUDES E COMPORTAMENTO PRÓ-AMBIENTAL}

Há evidências empíricas que dão suporte à relação entre valores, atitudes e comportamentos próambientais (ver, por exemplo, Milfont, Coelho Júnior, Gouveia \& Coelho, 2003; Schultz \& Zelezny, 1999; Vaske \& Donnelly, 1999). Rokeach (1968/1981) argumenta que as crenças, atitudes e valores estão atrelados, formando um sistema cognitivo funcionalmente integrado; portanto, uma mudança em qualquer parte deste sistema afetará outras partes e culminará em mudança comportamental.

Seguindo esta orientação, Stern e Dietz (1994) enfatizam que a orientação de valores pode afetar as crenças e atitudes dos indivíduos e, conseqüentemente, o seu comportamento. Partindo desta base teórica e ampliando o modelo de altruísmo (Schwartz, 1977), Stern e seus colaboradores (Stern \& Dietz, 1994; Stern, Dietz \& Kalof, 1993) propuseram que as normas morais podem ser ativadas não só por valores socioaltruísticos, mas também por valores egoístas e biosféricos. Stern e Dietz (1994) comprovaram empiricamente a correlação positiva dos comportamentos pró-ambientais com valores biosféricos, e negativa com valores egoístas.

Adicionalmente, Vaske e Donnelly (1999), com uma amostra de 960 participantes do Colorado (Estados Unidos da América), investigaram a operacionalização de um esquema de cognição hierárquica composta por: orientação valorativa $\rightarrow$ atitudes $\rightarrow$ intenção comportamental. Estes autores verificaram que a orientação valorativa (biocêntricalantropocêntrica) se apresenta como preditora de atitudes frente à preservação de florestas, e que estas medeiam a relação entre a orientação valorativa e a intenção comportamental de votar a favor da preservação das florestas. Neste estudo os indivíduos que apresentaram maior pontuação na orientação valorativa biocêntrica demonstraram uma atitude mais positiva com relação à preservação da floresta. Por outro lado, 
aqueles que apresentam uma orientação valorativa mais antropocêntrica tenderam a apoiar a expansão de áreas privadas de esqui sobre o espaço público próprio das florestas, mais do que o fizeram os indivíduos que apresentam uma orientação valorativa mais biocêntrica.

Karp (1996), em seu estudo sobre os valores e seus efeitos sobre o comportamento pró-ambiental, utilizou a tipologia proposta por Schwartz $(1992,1994)$ e verificou que, das quatro categorias de valores sugeridas por este autor, autotranscendência e abertura à mudança apresentaram influência positiva no comportamento pró-ambiental, enquanto autopromoção e conservação apresentaram direção inversa.

Schultz e Zelezny (1999) encontraram resultados semelhantes ao utilizar os tipos motivacionais de valores de Schwartz $(1992,1994)$ como preditores de atitudes ambientais. Mesmo considerando construtos diferentes, os resultados são comparáveis aos previamente descritos. Concretamente, estes autores verificaram que o tipo motivacional universalismo, que faz parte da categoria autotranscendência, foi o mais forte explicador das atitudes ambientais ecocêntricas. Mais detalhadamente, através de análises de regressão, estes autores verificaram que o tipo motivacional universalismo predisse positivamente as atitudes ecocêntricas, enquanto os tipos motivacionais poder $\mathrm{e}$ tradição o fizeram negativamente. Por outro lado, as atitudes antropocêntricas foram preditas positivamente pelos tipos motivacionais poder, tradição, conformidade e segurança, e negativamente, por benevolência.

Em resumo, estudos prévios têm demonstrado uma relação entre os valores humanos e as atitudes (tratadas predominantemente em termos das dimensões ecocêntrica e antropocêntrica) e comportamentos pró-ambientais; contudo, pouco se sabe efetivamente sobre o sentido e a magnitude com que estes construtos se correlacionam no contexto brasileiro, justificando a presente pesquisa.

\section{MÉTODO}

\section{Amostra}

Participaram desta pesquisa 208 estudantes de três cursos (Biologia, Filosofia e Psicologia) dos dois primeiros anos de duas universidades da Paraíba, sendo uma pública $(36,2 \%)$ e outra privada $(63,8 \%)$. A maioria destes era do sexo feminino $(73,4 \%)$, com idade média de 28 anos $(D P=7,0$; amplitude de 17 a
60). Os participantes indicaram, em sua maioria (73,2\%), pertencer à classe média.

\section{Instrumentos}

Questionário de valores de Schwartz: é composto de 56 valores-itens, e os indivíduos indicam numa escala de resposta que varia de $\mathbf{- 1}$ a $\mathbf{7}(\mathbf{- 1 , 0} \mathbf{0}, \mathbf{1 ,} \mathbf{2}, \mathbf{3}, \mathbf{4}$, $\mathbf{5 , 6 , 7 )}$ qual o grau de importância de cada valor (por exemplo, "união com a natureza") como um princípioguia na sua vida (Schwartz, 1992, 1994), sendo 0 = Não importante, $\mathbf{7}$ = Suprema importância e -1 = Oposto aos meus valores; os números de $\mathbf{1}$ a $\mathbf{6}$ indicam que quanto maior o número, mais importante é o valor como um princípio-guia. Informações sobre a sua validade de construto e consistência interna (do questionário de valores) podem ser obtidas em Tamayo e Schwartz (1993) e Gouveia, Martínez, Meira \& Milfont (2001).

Escala de atitudes ecocêntricas e antropocêntricas: foram utilizados 16 itens da escala de Thompson e Barton (1994), em sua versão revisada por Schultz e Zelezny (1999). Estes (itens) foram distribuídos para avaliar as atitudes antropocêntricas (por exemplo, "a razão mais importante pela qual devemos conservar a natureza é a sobrevivência dos seres humanos", "necessitamos conservar os recursos naturais para assegurara uma vida de alta qualidade") e ecocêntricas (por exemplo, "a natureza deve ser valorizada por si mesma”, “os seres humanos também fazem parte do sistema ecológico como qualquer outro animal"). Os participantes indicaram o seu grau de concordância ou discordância numa escala de cinco pontos, do tipo Likert, variando de $\mathbf{1}=$ Concordo totalmente a $\mathbf{5}=$ Discordo totalmente.

Além destes instrumentos, foram incluídas questões de natureza demográfica (idade, sexo e classe social) e uma solicitação que procurava avaliar a intenção de apresentar um comportamento próambiental. Especificamente, foi dito aos participantes que seria organizado um comitê para arrecadar dinheiro e realizar atividades em prol do meio ambiente para diminuir a contaminação e os desastres ecológicos, e que se necessitava de participação voluntária (evento fictício) de todos. Sendo assim, foilhes solicitado que, aqueles que se dispusessem a colaborar, deixassem seu nome ou apelido e telefone para contato.

\section{Procedimento}

Os instrumentos foram aplicados em salas de aula, utilizando-se um procedimento-padrão para garantir um mínimo de respostas enviesadas. Um único 
aplicador, com treinamento prévio, ficou responsável por dar as instruções aos participantes sobre como responder. Este interveio o mínimo possível no processo de aplicação, dando apenas explicações quando solicitadas e, evitando emitir significados ou conotações diferentes das atribuídas pelos participantes. Em média, 20 minutos foram suficientes para concluir esta atividade.

\section{Análise de dados}

Além de estatísticas descritivas, realizou-se uma análise de regressão linear com método enter para verificar quais dos dez tipos motivacionais de valores melhor explicariam as atitudes. Finalmente, efetuou-se uma análise de regressão logística com a finalidade de verificar quais dos construtos tratados melhor explicaria a intenção comportamental. Neste caso, o método forward stepwise (WALD) foi adotado.

\section{RESULTADOS}

Com a finalidade de testar a predição dos dez tipos motivacionais de valores humanos em relação às atitudes ambientais, realizou-se uma análise de Regressão Linear com o método enter. Os resultados desta análise são apresentados na Tabela 1.

Tabela 1. Regressão Linear Tendo os Valores como Preditores das Atitudes Ambientais

\begin{tabular}{|c|c|c|c|c|c|c|}
\hline \multirow[t]{2}{*}{ Valores } & \multicolumn{3}{|c|}{ Ecocentrismo } & \multicolumn{3}{|c|}{ Antropocentrismo } \\
\hline & B & Beta & $t$ & B & Beta & $t$ \\
\hline Benevolência & $-0,01$ & $-0,03$ & $-0,30$ & 0,05 & 0,07 & 0,71 \\
\hline Universalismo & 0,12 & 0,27 & $3,25 * *$ & $-0,02$ & $-0,03$ & $-0,47$ \\
\hline Estimulação & 0,02 & 0,03 & 0,33 & 0,06 & 0,05 & 0,56 \\
\hline Hedonismo & 0,07 & 0,05 & 0,58 & 0,22 & 0,11 & 1,26 \\
\hline Realização & $-0,06$ & $-0,10$ & $-1,05$ & 0,04 & 0,05 & 0,52 \\
\hline Autodireção & 0,02 & 0,05 & 0,63 & $-0,02$ & $-0,03$ & $-0,40$ \\
\hline Poder & 0,01 & 0,02 & 0,27 & 0,03 & 0,05 & 0,64 \\
\hline Segurança & $-0,05$ & $-0,10$ & $-0,98$ & 0,04 & 0,06 & 0,58 \\
\hline Tradição & 0,02 & 0,03 & 0,34 & 0,11 & 0,12 & 1,28 \\
\hline Conformidade & $-0,04$ & $-0,04$ & $-0,43$ & 0,05 & $-0,04$ & $-0,39$ \\
\hline \multirow[t]{3}{*}{ Constante } & 25,73 & & & \multicolumn{3}{|l|}{16,63} \\
\hline & \multicolumn{3}{|c|}{$R^{2}=0,07$} & $R^{2}=0,0$ & & \\
\hline & \multicolumn{3}{|c|}{$F(10,186)=1,39$} & $F(10,18$ & )$=1,92$ & \\
\hline
\end{tabular}

Nota: $* * p<0,01$. O valor estatisticamente significativo do Beta é apresentado em negrito.

Verificou-se que o tipo motivacional de valores universalismo foi consistentemente o que melhor explicou o ecocentrismo. Considerando as quatro dimensões bipolares de valores - abertura à mudança versus conservação e autopromoção versus autotranscendência - como explicadoras das atitudes ambientais, realizou-se uma análise de Regressão
Linear com o método enter. Os resultados podem ser vistos na Tabela 2. Dentre as quatro, verificou-se que a dimensão autotranscendência foi a que melhor explicou o ecocentrismo.

Tabela 2. Regressão Linear Tendo as Quatro Categorias de Valores como Preditoras das Atitudes Ambientais

\begin{tabular}{lcccccc}
\hline Valores & \multicolumn{3}{c}{ Ecocentrismo } & \multicolumn{3}{c}{ Antrocentrismo } \\
\cline { 2 - 7 } & $\mathbf{B}$ & Beta & $\boldsymbol{t}$ & $\mathbf{B}$ & Beta & $\boldsymbol{t}$ \\
\hline Abertura à mudança & 0,03 & 0,07 & 0,94 & 0,03 & 0,07 & 0,91 \\
Conservação & $-0,04$ & $-0,14$ & $-1,43$ & 0,05 & 0,15 & 1,47 \\
Auto-promoção & $-0,01$ & $-0,05$ & $-0,52$ & 0,05 & 0,10 & 1,11 \\
Auto-transcendência & $\mathbf{0 , 0 7}$ & $\mathbf{0 , 2 4}$ & $\mathbf{2 , 7 2 * *}$ & $-0,00$ & $-0,00$ & $-0,08$ \\
Constante & 24,91 & \multicolumn{5}{c}{17,11} \\
\hline & $R^{2}=0,04$ & \multicolumn{3}{c}{$R^{2}=0,07$} \\
& $F(4,196)=2,231$ & \multicolumn{3}{c}{$F(4,191)=3,57$} \\
\hline
\end{tabular}

Nota: $* * p<0,01$. O valor estatisticamente significativo do Beta é apresentado em negrito.

Por fim, com o intuito de verificar em que medida os valores e as atitudes ambientais permitem explicar o compromisso de se engajar em alguma atividade em favor do meio ambiente, realizou-se uma análise de Regressão Logística com o método Forward Stepwise $(W A L D)$. Os resultados indicaram que os valores que compõem o tipo motivacional universalismo foram os mais adequados para explicar o compromisso de se engajar em alguma atividade em favor do meio ambiente, $R^{2}=0,07$ [Wald $(1)=7,85, p<0,05$ ].

\section{DISCUSSÃO}

O objetivo desta pesquisa foi conhecer o papel dos valores humanos como explicadores das atitudes e intenção comportamental pró-ambiental no contexto brasileiro. Três limitações do estudo, no entando, precisam ser enunciadas. Em primeiro lugar, utilizou-se uma medida de auto-relato (self-report) para avaliar a intenção comportamental. Embora amplamente utilizada, esta estratégia de coleta de dados tende a superestimar comportamentos pró-ambientais. Hines e cols. (1987) verificaram que a forma de mensuração destes comportamentos (através de verificação do comportamento real ou através de auto-relatos) atenua a correlação entre atitudes e comportamento; assim, as correlações são maiores quando o comportamento real é mensurado. Por isso, recomenda-se o uso de outros métodos, tais como a observação direta ou os traços de comportamento (Corral-Verdugo \& Pinheiro, 1999).

Outra limitação diz respeito ao instrumento utilizado para avaliar as atitudes ambientais. Este apresentou índices meritórios de consistência interna, com Alfas de 0,54 e 0,63 para os fatores 
antropocentrismo e ecocêntrismo, respectivamente. Com estas duas dimensões apresentam respaldo teórico e empírico (Kortenkamp \& Moore, 2001; Thompson \& Barton, 1994; Vaske \& Donnelly, 1999), recomenda-se a construção de mais e melhores itens que abarquem os construtos em questão. Por fim, neste estudo foram considerados apenas estudantes universitários, cumprindo salientar que a maioria é do sexo feminino, o que pode ter influenciado os resultados encontrados. Como as pessoas mais jovens tendem a apresentar mais procupação ambiental (Fransson \& Gärling, 1999), recomenda-se a replicação desta pesquisa com amostras da população geral, não-estudante.

Embora apresente estas limitações, a pesquisa cumpriu os objetivos a que se propôs, verificando as relações entre os valores humanos, as atitudes ambientais e a intenção de apresentar comportamento pró-ambiental no contexto brasileiro. Verificou-se, também, que o tipo motivacional universalismo foi consistentemente o que melhor explicou ecocentrismo, corroborando o que tem sido observado em estudos anteriores (Karp, 1996; Schultz \& Zelezny, 1999). Karp (1996) verificou, por exemplo, que das quatro categorias de valores sugeridas por Schwartz (1992, 1994), autotranscendência e abertura à mudança foram fortes preditores do comportamento próambiental. Verificou-se que as pessoas que pontuaram mais fortemente na dimensão autotranscendência apresentaram em maior gradiente atitudes ecocêntricas e o compromisso em participar de atividades em favor do meio ambiente.

O tipo motivacional universalismo representa compreensão, apreço, tolerância e proteção do bemestar dos indivíduos e da natureza. Assim, pessoas que assumem esta orientação valorativa tendem a apresentar atitudes, crenças e compromissos em favor do meio ambiente em maior medida do que aqueles que não priorizam tais valores. Concretamente, verificou-se que as pessoas que pontuaram mais alto em universalismo apresentaram mais predisposição a agir de modo que favorecesse o ambiente.

Levando-se em conta ainda o argumento de Stern e Dietz (1994), de que a orientação de valores pode afetar as crenças e atitudes dos indivíduos e, conseqüentemente, o seu comportamento, o estudo dos valores pode contribuir consistentemente para a modificação de condutas de descaso ambiental.

Desse modo, a educação de valores que contemplem a dimensão universalismo (justiça social, sabedoria, igualdade, um mundo em paz, harmonia interior, um mundo de beleza, união com a natureza, protetor do ambiente e aberto) pode favorecer o desenvolvimento de atitudes ecocêntricas e, conseqüentemente, comportamentos pró-ambientais. Esses valores podem ser ensinados já no ensino fundamental, procurando socializar as crianças em princípios que destaquem a preservação e promoção do meio ambiente. A presente pesquisa representa potencial subsídio para qualquer iniciativa de impacto no âmbito das políticas públicas e dos projetos voltados ao incentivo do desenvolvimento de condutas próambientais da população brasileira, a ser concebida e planejada por agentes da iniciativa privada, dos órgãos vinculados aos poderes públicos e do chamado terceiro setor.

Enfim, o estudo de atitudes e valores capaz de assegurar um ambiente sustentável deveria ser uma meta principal dos pesquisadores em Psicologia Social e Ambiental (Schmuck \& Schultz, 2002). Portanto, novos estudos são demandados. Exemplo: poder-se-ia estudar a adequação da hierarquia valores $\rightarrow$ atitudes $\rightarrow$ comportamento. Esta tem sido assumida (Rokeach, 1968/1981), porém são ainda escassos os estudos em que foi avaliada em relação à preocupação ambiental (Vaske \& Donnelly, 1999). Seria útil pensar na elaboração de instrumentos emics, procurando avaliar, por exemplo, a preocupação frente à preservação da fauna, flora e dos recursos minerais brasileiros, há muito ameaçados (Elston, 2001; Oskamp, 2000).

\section{REFERÊNCIAS}

Almeida Júnior, J. M. G. (1994). Desenvolvimento ecologicamente auto-sustentável: conceitos, princípios e implicações. Humanidades, 10, 284-299.

Ajzen, I. \& Fishbein, M. (1985). Understanding attitudes and predicting social behavior. Englewood Cliffs: PrenticeHall.

Albrecht, D., Bultena, G., Hoiberg, E. \& Nowak, P. (1982). The new environmental paradigm scale. The Journal of Environmental Education, 13, 39-42.

Alves Filho, F. (2002, 03 de abril). País sujo. Revista Isto É, 1696, 74-80.

American Psychological Association. (2001, 9th Ed.). Thesaurus of psychological index terms. Washington: American Psychological Association.

Bechtel, R. B., Verdugo, V. C. \& Pinheiro, J. Q. (1999). Environmental belief systems: United States, Brazil and Mexico. Journal of Cross-cultural Psychology, 30, 122128.

Cameron, L. D., Brown, P. M. \& Chapman, J. G. (1998). Social value orientations and decisions to take proenvironmental action. Journal of Applied Social Psychology, 28, 675-697.

Cortez, J. C. V., Milfont, T. L. \& Belo, R. P. (2001). Significados psicológicos do lixo: um estudo através das redes semânticas naturais. Psico-USF, 6, 21-28. 
Corral-Verdugo, V. (2000). La definición del comportamiento proambiental. En La Psicología Social en México, La Psicología Social en México (Vol. VIII, pp. 466-472). Guadalajara: MAEPSO - Asociación Mexicana de Psicología Social.

Corral-Verdugo, V. \& Pinheiro, J. Q. (1999). Condições para o estudo do comportamento pró-ambiental. Estudos de Psicologia, 4, 7-22.

Dunlap, E. R. \& Van Liere, K. D. (1978). The "New Environmental Paradigm". Journal of Environmental Education, 9, 10-19.

Dutra, E. E. J. \& Tamayo, A. (2000). Prioridades axiológicas e percepção do trabalho de equipe. [Resumo]. Em Sociedade Brasileira de Psicologia (Org.), Resumos de comunicações científicas. XXX Reunião Anual de Psicologia (p. 212). Brasília: SBP.

Elston, S. (2001). Time running out on environment, report says. Environmental News Network. Disponível em: $<$ http://www.enn.com/news/enn-

stories/2001/01/01182001/_enviroforecast_41407.asp> (Acesso em 29/06/2004).

Fransson, N. \& Gärling, T. (1999). Environmental concern: Conceptual definitions, measurement methods, and research findings. Journal of Environmental Psychology, 19, 369-382.

García-Mira, R., Real-Deus, E. \& Romay-Martínez, J. (2000). Predicción de actitudes y comportamiento ambiental. En A. O. Bernal, M. V. M. Jiménez \& P. V. Elias (Orgs.), Aplicaciones em psicología social (pp. 354-361). Madri: Biblioteca Nueva.

Gouveia, V. V. (2002). Self, culture and sustainable development. In P. Schmuck \& W. P. Schultz (Eds.), Psychology of sustainable development (pp. 151-174). Norwell, Mass.: Kluwer Academic Publishers.

Gouveia, V. V., Martínez, E., Meira, M. \& Milfont, T. L. (2001). A estrutura e o conteúdo universais dos valores humanos: Análise fatorial confirmatória da tipologia de Schwartz. Estudos de Psicologia, 6, 133-145.

Hernández, B. \& Hidalgo, M. C. (1998). Actitudes y creencias hacia el medio ambiente. En J. I. Aragonés \& M. Amérigo (Orgs.), Psicología ambiental (pp. 281-295). Madri: Pirámide.

Hines, J. M., Hungerford, H. R., \& Tomera, A. N. (1987). Analysis and synthesis of research on responsible environmental behavior: a meta-analysis. Journal of Environmental Education, 18, 1-8.

Hinrichsen, D. \& Robey, B. (2000). Population and the Environment: The global challenge. Population Reports, 8 Disponível <http://www.infoforhealth.org/pr/m15edsum.shtml>. (Acesso em 29/06/2004).

Howard, G. S. (2000). Adapting human lifestyles for the 21st century. American Psychologist, 55, 509-515.

Kaiser, F. G. (1998). A general measure of ecological behavior. Journal of Applied Social Psychology, 28,395422.

Kaiser, F. G., Ranney, M., Hartig, T. \& Bowler, P. A. (1999). Ecological bahavior, environmental attitude, and feelings of responsibility for the environment. European Psychologist, 4, 59-74.

Karp, D. G. (1996). Values and their effect on proenvironmental behavior. Environment \& Behavior, 28, 111-133.

Kidner, D. (2001). Nature and psyche: Radical environmentalism and the politics of subjectivity. New York: State University of New York Press.

Kortenkamp, K. V. \& Moore, C. F. (2001). Ecocentrism and anthropocentrism: Moral reasoning about ecological commons dilemmas. Journal of Environmental Psychology, 21, 261-272.

Maloney, M. P. \& Ward, M. P. (1973). Ecology: Let's hear it from the people - An objective scale for measurement of ecological attitudes and knowledge. American Psychologist, 28, 583-586.

McKenzie-Mohr, D. (2000). Fostering sustainable behavior through community-based social marketing. American Psychologist, 55, 531-537.

Milfont, T. L., Coelho Júnior, L. L., Gouveia, V. V. \& Coelho, J. A. P. M. (2003, set.). Human values: Their correlation with environmental attitudes and behaviours. Trabalho apresentado em Reunião Bianual ( $5^{\text {th }}$ Biannual Meeting) da Division of Environmental Psychology of the German Psychological Association, Eindhoven, The Netherlands.

Oskamp, S. (2000). A sustainable future for humanity? How can psychology help? American Psychologist, 55, 496-508.

Rodrigues, A., Assmar, E. M. L. \& Jablonski, B. (1999). Psicologia Social. Petrópolis: Vozes.

Rokeach, M. (1981). Crenças, atitudes e valores. Rio de Janeiro: Interciência. (Original publicado em 1968).

Schmuck, P. \& Schultz, W. P. (2002) (Eds.). Psychology of sustainable development. Norwell: Kluwer Academic Publishers.

Schultz, P. W. (2002). Inclusion with nature. In P. Schmuck \& W. P. Schultz (Eds.), Psychology of sustainable development (pp. 61-78). Norwell: Kluwer Academic Publishers.

Schultz, P. W. \& Zelezny, L. (1999). Values as predictors of environmental attitudes: Evidence for consistency across 14 countries. Journal of Environmental Psychology, 19, 255-265.

Schultz, P. W., Zelezny, L. C. \& Dalrymple, N. J. (2000). A multinational perspective on the relation between JudeoChristian religious beliefs and attitudes of environmental concern. Environment \& Behavior, 32, 576-591.

Schwartz, S. H. (1977). Normative influences on altruism. In L. Berkowitz (Ed.), Advances in experimental social psychology, (Vol. 10, pp. 221-271). New York: Academic Press.

Schwartz, S. H. (1992). Universals in the content and structure of values: Theoretical advanced and empirical tests in 20 countries. In M. Zanna (Ed.), Advanced in experimental social psychology (pp. 1-65). Orlando: Academic Press. 
Schwartz, S. H. (1994). Are there universal aspects in the structure and contents of human values? Journal of Social Issues, 50, 19-45.

Schwartz, S. H. \& Bilsky, W. (1987). Toward a universal psychological structure of human values. Journal of Personality and Social Psychology, 53, 550-562.

Schwartz, S. H. \& Bilsky, W. (1990). Toward a theory of the universal content and structure of values: Extensions and cross-cultural replications. Journal of Personality and Social Psychology, 58, 878-891.

Stern, P. C. (2000). Psychology and the science of humanenvironment interactions. American Psychologist, 55, 523530.

Stern, P. C. \& Dietz T. (1994). The value bases of environmental concern. Journal of Social Issues, 50, 6584.

Stern, P. C., Dietz T. \& Kalof L. (1993). Value orientations, gender, and environmental concern. Environmental and behavior, 25, 322-348.

Tamayo, A. (1994). Hierarquia de valores transculturais e brasileiros. Psicologia: Teoria e Pesquisa, 10, 269-285.

Tamayo, A. (1997). Os valores do brasileiro: uma década de pesquisa. Cadernos de Psicologia, 1, 115-134.

Tamayo, A. (2001). As prioridades axiológicas como preditoras da exaustão emocional no trabalho [Resumo]. Em Sociedade Brasileira de Psicologia (Org.), Resumos de comunicações científicas. XXXI Reunião Anual de Psicologia (p. 98). Rio: SBP.

Tamayo, A., Lima, A., Marques, J., \& Martins, L. (2000). Os valores pessoais como preditores do uso de preservativo. [Resumo]. Em Sociedade Brasileira de Psicologia (Org.), Resumos de comunicações científicas. XXX Reunião Anual de Psicologia (pp. 45-46). Brasília: SBP.

Tamayo, A., Lima, G. L., Siqueira, K. D. M., Vargas, K. C., Salomão, M. A. \& Rodrigues, W. A. (2000). Exaustão emocional: A profissão e os valores pessoais como preditores. [Resumo]. Em Sociedade Brasileira de Psicologia (Org.), Resumos de comunicações científicas. XXX Reunião Anual de Psicologia (pp. 217-218). Brasília: SBP.

Tamayo, A., Pereznebra, A. R., Zandonadi, L. P. \& Benjamin, M. S. (2002). Prioridades axiológicas como preditores de exaustão emocional. [Resumo]. Em Sociedade Brasileira de Psicologia (Org.), Resumos de comunicações científicas. XXXII Reunião Anual de Psicologia (pp. 314315). Florianópolis: SBP.

Tamayo, A. \& Schwartz, S. H. (1993). Estrutura motivacional dos valores humanos. Psicologia: Teoria e Pesquisa, 2, 329-348.

Tamayo, A., Souza, M. G. S., Vilar, L. S., Ramos, J. L., Albenaz, J. V. \& Ferreira, N. P. (2001). Prioridades axiológicas e comprometimento organizacional. Psicologia: Teoria e Pesquisa, 17, 27-35.

Tamayo, A., Zandonadi, L. P., Nepomuceno, M. V., Costa Filho, J. B. \& Barros, C. C. (2000). Comparação dos valores de presos e de agentes penitenciários. [Resumo]. Em Sociedade Brasileira de Psicologia (Org.), Resumos de comunicações científicas. XXX Reunião Anual de Psicologia (pp. 272-273). Brasília: SBP.

Thompson, S. C. G. \& Barton, M. A. (1994). Ecocentric and anthropocentric attitudes toward the environment. Journal of Environmental Psychology, 14, 149-157.

Vaske, J. J. \& Donnelly, M. P. (1999). A value-attitudebehavior model predicting wildland preservation voting intentions. Society \& Natural Resources, 12, 523-537.

Weigel, R. H. \& Newman, L. S. (1976). Increasing attitudebehavior correspondence by broadening the scope of the behavioral measure. Journal of Personality and Social Psychology, 33, 793-802.

Weigel, R. \& Weigel, J. (1978). Environmental concern: The development of a measure. Environment and Behavior, 10, 3-15.

Zandonadi, L. P., Pérez, A. R. \& Tamayo, A. (2001). Valores laborais e comprometimento organizacional. [Resumo]. Em Sociedade Brasileira de Psicologia (Org.), Resumos de comunicações científicas. XXXI Reunião Anual de Psicologia (pp. 254-255). Rio: SBP.

Recebido em 23/08/2004 Aceito em 30/09/2005

Endereço para correspondência: Valdiney V. Gouveia. Universidade Federal da Paraíba, CCHLA, Departamento de Psicologia, CEP 58059-901, João Pessoa-PB. E-mail: vvgouveia@uol.com.br 